EOMmUn Communication et organisation

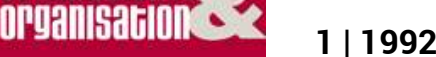

Communication et sentiment d'appartenance

\title{
Images de soi et organisation
}

Jean-Jacques Boutaud

\section{OpenEdition}

Journals

Édition électronique

URL : http://journals.openedition.org/communicationorganisation/1540

DOI : 10.4000/communicationorganisation. 1540

ISSN : 1775-3546

Éditeur

Presses universitaires de Bordeaux

Édition imprimée

Date de publication : 1 mai 1992

ISSN : 1168-5549

Référence électronique

Jean-Jacques Boutaud, «Images de soi et organisation », Communication et organisation [En ligne], 1 | 1992, mis en ligne le 26 mars 2012, consulté le 30 avril 2019. URL : http://journals.openedition.org/ communicationorganisation/1540 ; DOI : 10.4000/communicationorganisation.1540

Ce document a été généré automatiquement le 30 avril 2019

(c) Presses universitaires de Bordeaux 


\title{
Images de soi et organisation
}

\author{
Jean-Jacques Boutaud
}

1 Dans les termes complexes où il faut penser la vie en organisation, le sentiment d'appartenance n'est pas la moindre des questions à élucider. Dans son origine, sa forme et ses manifestations, dans ses effets aussi. Cette question s'ouvre à un cadre épistémologique non moins complexe. Le sentiment d'appartenance convoque bien entendu des notions psychologiques, philosophiques, psychanalytiques mais aussi sociologiques, anthropologiques, culturelles, sans épuiser pour autant le sujet.

De cette diversité naît la richesse du thème dont nous proposons ici une analyse en termes de communication. Tout le fond de cette étude est redevable à l'économie signifiante de ce discours pour rendre compte du système de signification en jeu dans le sentiment d'appartenance. À commencer par l'image de l'organisation qui offre prise à ce sentiment. Qu'en est-il aujourd'hui, dans son rapport à l'individu, de l'eschatologie d'entreprise, comprise ici dans son regroupement le plus large avec les services et les administrations? Comment se mesure, dans les formes du discours et de l'organisation, la place de l'homme? Les contingences de sa situation sont-elles ou non propices, selon les cas, à entretenir le sentiment d'identité avec le groupe?

3 Face à ces interrogations liminaires, à développer, il faut poser les modèles de référence de la construction, de la conceptualisation de l'image de soi, telle que l'individu peut la représenter dans la relation au groupe. Comment répondre alors aux critères d'appartenance définis par ce groupe? Reconnaître ces critères est-ce aussi se reconnaître, au gré d'un processus gratifiant ou non?

4 Autant de questions qu'il nous est impossible d'ajourner dans la recherche des points d'articulation où le sentiment d'appartenance prend sa signification. La relation primitive entre l'image de l'organisation et l'image de soi génère, en l'occurrence, d'autres relations signifiantes à intégrer, pour les expliciter, dans un champ de vision élargi. Globalement il s'agira d'étudier un système où s'inter-définissent les modalités d'être constitutives du schème d'appartenance au groupe.

5 Appartenir à une organisation ne suffit pas à déclencher le sentiment d'appartenance. Encore faut-il, pour encourager cette relation d'identité gratifiante au groupe, favoriser chez l'individu les conditions d'une prise de conscience sur son rôle, sa situation. À cet 
égard, la nature évolutive des conceptions traverse l'histoire de l'entreprise, en donnant au sentiment d'appartenance différents modèles de référence. Pour fixer les idées et situer la place actuelle de l'individu dans l'organisation, le rapport d'intersubjectivité qui les unit, retenons quelques étapes marquantes.

6 Sans remonter à la préhistoire de l'entreprise, on retiendra, pour leur contemporanéité au début de ce siècle, deux modèles de l'utilitarisme libéral. L'un en France, avec Fayol ; l'autre aux États-Unis, avec Taylor. La place dévolue à l'homme, à l'intérieur des deux systèmes préconisés, comporte de lourdes hypothèques sur les possibilités d'épanouissement de l'individu, sans pour autant gommer sa caution virtuelle à de tels systèmes.

7 En résumé, les éléments qui intéressent notre propos viennent déjà d'un ingénieur français, Fayol, porté dès 1916 à définir de nouvelles règles pour l'administration des entreprises. Son ouvrage sur l'Administration industrielle et générale ${ }^{1}$ déborde la formule célèbre : "diriger c'est prévoir, organiser, commander, coordonner, contrôler », pour poser les bases d'une bonne gestion du personnel. Pour cela, il faut éduquer, c'est-à-dire affirmer la nécessité d'une organisation hiérarchique des diverses fonctions (administrative, technique, commerciale, financière, comptable) et mettre les ouvriers en situation de comprendre, entre autres, les lois du marché. De là, pour reprendre l'expression d'A. Smith, leur intérêt "bien compris» pour une politique patronale rationnelle. En prime aussi la stabilité sous l'égide d'un pouvoir auquel chacun s'en remet individuellement, dans l'intérêt collectif.

8 Avec le modèle taylorien, beaucoup plus répandu depuis les États-Unis, la mécanique utilitaire s'impose encore davantage à la réalité du travail. Dans la logique bien huilée du darwinisme social «l'homme de première classe » trouve, par le travail, l'affirmation de sa dignité partagée (le pacte social ouvrier-patron), de son élévation morale (la journée de travail «loyale»). Ainsi se cristallise, dans La Direction Scientifique des Entreprises², l'imaginaire libéral et utilitariste où chacun aurait à gagner. Notamment les ouvriers grâce aux fameuses primes de rendement, garantie de leur efficacité mais aussi de leur équité, de la fidélité promise au pouvoir transcendant et à son organisation. Pour autant le sentiment d'appartenir à cet ordre "naturel » n'a conduit, par sa simplification et la spécialisation à outrance avec la machine, qu'à déshumaniser le travail. Inutile de reprendre ici les conclusions des contempteurs de ce "travail en miettes $»^{3}$. Cependant bien avant l'usure du modèle, des expériences ont conduit à repenser la situation de l'homme au travail. Notamment au sein d'organisations dont il pourrait se sentir solidaire, sur des bases conscientes et confiantes.

9 L'expérience sans conteste la plus marquante se déroule dans le berceau même du taylorisme, aux États-Unis. Plus exactement dans l'usine de la société Western Electric à Hawthorne, dans l'Illinois. Les travaux d'Elton Mayo, publiés en $1933^{4}$ sont guidés, dans un premier temps, par la recherche des conditions possibles d'amélioration des performances, chez les ouvrières opérant sur une chaine de montage. Le rapport à la rentabilité se veut ainsi calculé selon l'introduction de variables comme, par exemple, la lumière ou le temps de travail dans le cadre expérimental. Les résultats non seulement surprennent mais opèrent un renversement des vues tayloriennes, du mythe de l'homo economicus. En effet, l'expérience montre que, bonnes ou mauvaises, les conditions de travail vécues par le groupe expérimental augmentent sa rentabilité. La preuve est faite selon Mayo - ses conclusions joueront un rôle capital dans l'émergence des Relations Humaines - qu'au-delà du salaire, de l'argent, l'appartenance à un groupe social, avec ses 
normes, son rôle de soutien, représente un facteur fondamental de la vie au travail. Voilà comment expliquer la réaction des ouvrières, encouragées, dans le contexte expérimental, à une prise de conscience de leur rôle, à des relations de groupe amicales et informelles; ouvrières sensibles aussi à la prise en considération de leur existence par la Direction, avec une attention supposée au concours ainsi apporté. La leçon de l'expérience Hawthorne retentit jusqu'au cœur du discours actuel en communication A savoir que l'individu ne réagit pas aux conditions physiques de l'environnement en situation objective mais telles qu'il les ressent. Cela constitue aujourd'hui l'un des fondements de l'analyse systémique dont il sera question plus loin.

10 À ce stade, retenons de l'évolution des organisations, le passage du discours utilitaire à des valeurs non utilitaires, avec progressivement l'homme comme valeur ajoutée. Au premier rang des préoccupations, le pouvoir d'intégration de l'homme dans l'organisation, son degré de mobilisation à l'intérieur des systèmes et sous-systèmes, son besoin de réalisation en conformité avec le groupe de référence.

11 Cette démarche se trouve encore très liée, de nos jours, à l'apport de Kurt Lewin, le fondateur de la dynamique des groupes ${ }^{5}$. L'analyse de la personnalité de l'individu dans son environnement fait entrer, dans sa modélisation, le concept de " champ ", repris par Lewin à la théorie de la Gestalt. Les conduites humaines se conçoivent alors dans un champ de forces psychologiques et individuelles mais aussi dans le champ social des forces internes au groupe. Ce dernier procède de sa propre dynamique au lieu d'apparaître comme une entité mue de l'extérieur. La composition des forces, à valence négative (contrainte) et positive (gratification) crée le champ de motivation nécessaire à l'individu pour se reconnaître dans son groupe, s'identifier à son action, sans recours obligé à une autorité extérieure. Dans le système des interdépendances produites par le groupe, d'une part entre individus, d'autre part entre éléments du champ (normes, modèles, représentations), se font jour des paramètres comme le moral, la motivation, le feed-back, le leadership. Faire partie d'un groupe c'est alors faire sens par l'action pour devenir agent du changement social, à l'intérieur de secteurs toujours plus vastes et bientôt en interaction avec le système. Voilà jetées les bases de l'école dite des Relations Humaines.

12 Restent à approfondir les relations entre l'Acteur et le Système ${ }^{6}$ pour continuer dans cette voie de réhabilitation de l'individu. On aurait tort, en effet, de jeter un regard naïf sur le courant des Relations Humaines, non dénué d'arrière-pensées en termes de productivité, de rentabilité, de profit dont la communication interpersonnelle serait le lubrifiant. Bien sûr le salarié gagne à se situer dans un champ de forces dynamiques, à développer en interdépendance un rayon d'action toujours plus grand; globalement il gagne à vivre dans et par un environnement ouvert à tous les points de fixation professionnelle, psychologique, sociale, culturelle. Attention toutefois à faire de ce sentiment d'appartenance la garantie de sa liberté et non de sa domesticité, de son conditionnement à des modèles d'acculturation maison. Pour être un progrès, la vogue des Relations Humaines n'invite pas moins à admettre « la validité du principe du « one best way » pour tout ce qui concerne la rationalité technique et organisationnelle qu'elle ne peut mettre en cause » et, pour suivre la mise en garde de Michel Crozier, " elle crée un nouveau risque sur lequel ont buté à la fois des théoriciens et des praticiens des Relations Humaines, le risque de manipulation... On ne peut échapper à cette tentation que si l'on reconnaît que l'homme n'est pas seulement une main et un cœur, mais qu'il est aussi une tête, c'est-à-dire un agent autonome et libre, capable de réagir non seulement contre la 
standardisation taylorienne mais aussi contre la manipulation psychologique $»^{7}$. Il sera question plus loin d'adopter cet angle d'analyse. Mais pour en faciliter l'éclairage, passons à un autre registre de considérations. Une fois acquis le principe de reconnaissance de l'individu dans l'organisation, une fois perçues les conditions d'émergence d'un sentiment d'identification avec l'environnement interne, voyons comment l'image même de cet environnement entretient ce sentiment.

Dans une société en perte de vitesse idéologique, en crise de modèles eschatologiques, les entreprises s'affirment comme les derniers territoires à pouvoir répondre à la quête identitaire du sujet. Ce qu'elles offrent? Une culture, des valeurs et surtout un cadre mythologique où le monde du travail se libère des contingences pour parler à l'imaginaire. Le discours du patron fait place au métadiscours de l'entreprise se donnant, sur le mode anthropomorphique, une image mythique et glorifiante. Son terrain est un champ de bataille où il faut apprendre à défendre ses couleurs (le groupe, la France, l'Europe), à lutter (contre les concurrents, les menaces internes et externes), à vaincre (seuls les plus forts doivent survivre pour l'intérêt commun). Au paradigme de la guerre sauvage, primaire, vient se substituer l'image de la compétition. Les mots résonnent à l'unisson comme un acte de bravoure: contrat, défi, challenge. Les vainqueurs entrent dans un palmarès, se voient décerner des trophées. Forts de cette image ils peuvent parader, porter haut leurs couleurs. Ils conquièrent de nouveaux territoires comme le sport, les arts, les lettres. Signes d'une légitimité acquise, d'une philosophie éclairée. En un mot, signes d'une culture. Ne raillons pas car voilà que se dressent de nouveaux héros comme autant de modèles promis à notre imaginaire. Dans le concert des grandes nations ils ressortent comme l'incarnation du prestige, de la réussite, du pouvoir. Leur nom s'inscrit dans la légende : Iacocca (Chrysler, États-Unis); Morita (Sony, Japon); pour la France, l'incontournable figure de Bernard Tapie. Comment résister au charme de leur discours sur l'entreprise qui gagne? Comment ne pas se laisser porter par l'euphorie d'une victoire promise?

14 Entrer dans ce monde d'élus implique, au minimum, de donner le change, à des niveaux de considération proportionnels aux responsabilités. Les degrés d'exigence, on le devine, se corrigent en fonction du statut et du rôle recherché par l'individu au sein de l'organisation. Depuis la plus simple tâche d'exécution jusqu'au pouvoir de direction sur des opérations définies. Mais, comme il ressort du premier volet de notre étude, l'émergence des Relations Humaines suppose la mobilisation de tous les acteurs à l'intérieur du système. La vie en entreprise fait de la relation au groupe un critère fondamental de son évolution.

15 En première approximation, la fameuse pyramide de Maslow $^{8}$ place le sentiment d'appartenance en position intermédiaire entre la satisfaction des besoins primaires (en termes physiologiques comme au plan de la sécurité), et les deux besoins supérieurs que sont la reconnaissance et surtout l'accomplissement. Maslow insiste notamment sur l'homéostasie nécessaire des trois premiers niveaux (physiologie, sécurité, appartenance) pour viser les degrés de réalisation les plus nobles, sans espoir de les satisfaire définitivement. On aurait tort, bien sûr, de s'en tenir à une interprétation fixiste de cette échelle à laquelle les réactions particulières des individus apportent parfois le démenti. Ainsi le salarié de base ne revendique pas moins sa dignité, sa reconnaissance dans le groupe, quand bien même il ressent quotidiennement le poids des exigences primaires (faire un boulot, s'y accrocher). Mais gardons-nous également d'une vision naïve du problème. Le sentiment d'appartenance trouve bien son origine dans une relation 
d'ouverture à l'environnement interne et externe, ce qui place en position favorable les individus susceptibles ou capables d'exprimer des opinions, de prendre des décisions. La finalité des cercles de qualité s'inscrit tout entière dans ce projet de communication globale, sans effacer la discrimination des rôles, ni cultiver à égalité le pouvoir d'identité avec l'âme du groupe.

Une tendance se dessine néanmoins pour mesurer, dès l'embauche, et stimuler par la suite, le degré de reconnaissance mutuelle entre le sujet et l'organisation. À cet égard si l'entreprise fait l'effort de promouvoir son image sur le versant non utilitaire des valeurs humaines, il est en retour demandé à l'individu de s'interroger sur sa propre image. L'affirmation d'une identité, d'une personnalité, sur un mode spontané, ne suffisent pas à rendre compte du degré de raffinement atteint aujourd'hui dans la connaissance d'un moi légitime aux yeux de l'organisation. Les grilles d'analyse se multiplient, les systèmes interprétatifs se chevauchent, au point de donner une vigueur sans précédent à la vieille maxime philosophique: "nosce te ipsum» (connais-toi toi-même). Appartenir à un groupe, être en situation de s'y reconnaître pose, comme jamais dans notre histoire, un préalable essentiel : se connaître soi-même.

ection de son image conditionne déjà l'accès à l'organisation. Le processus en jeu ne vise pas un moi idéal, affirmant sa toute puissance narcissique, mais à proprement parler une image de soi conforme aux modèles de référence conventionnels. C'est-à-dire les modèles promus par notre société, relayés par le discours économique dominant et actualisés par les valeurs de prestige de l'entreprise communicante. À charge pour l'individu de se retrouver dans la correspondance naturelle ou non à ces modèles dominants. Le passage de l'homo economicus à l'homo communicans marque dans le même temps une coupure épistémologique entre le paradigme de l'homme unidimensionnel (Marcuse) et le nouveau modèle de l'homme multidimensionnel (J. de Rosnay). Cette notion explicite le besoin d'identité du sujet à des niveaux d'évaluation complémentaires. L'expression d'une auto-référence et sa modélisation professionnelle s'articulent autour des « quatre composantes fondamentales suivantes : biologique (dont l'unité est l'organisme) ; intellectuelle et comportementale (dont l'unité est la personne) ; sociale et relationnelle (dont l'unité est le citoyen) et symbolique (dont l'unité est l'être). Ces quatre composantes s'intègrent dans la totalité que représente l'homme unidimensionnel $»^{9}$. Reprenons une à une ces composantes dont l'expression narcissique fonde le sentiment de participation aux rituels de la vie collective et partant, au discours institutionnel du groupe.

D'abord au plan biologique. L'impératif premier de l'équilibre, le sujet se trouve en situation d'y souscrire par l'action de deux forces antagonistes. D'une part l'affirmation confiante d'un capital d'énergie "à revendre ", d'autre part la mise au défi de répondre à des charges professionnelles aussi accaparantes que possibles. Si le terme de ce contrat garantit sens et reconnaissance, la motivation joue à plein. Dans la relation à l'autre, elle fixe un objet à la quête de soi. Ces dispositions profondes, philosophiques par essence, biologiques par leurs mécanismes d'auto-régulation, prennent en surface les traits physiologiques de l'aisance, de la plénitude corporelle. Les marques naturelles gagnent à s'afficher : de la beauté à la séduction pour les plus favorisés; de la santé à la vitalité, pour les mieux disposés. Prédicats physiques et psychiques, corrélés dans l'expression de la personne, forgent non seulement l'image de la maîtrise de soi mais encouragent, au besoin, toutes les figures du dépassement. Ainsi faut-il interpréter les rituels de compétition et les défis sportifs que se lancent les professions (courses, marathons, saut à 
l'élastique, en parachute), où se condensent la métaphore et la réalité vivante. Le besoin biologique rejoint la quête symbolique avec le désir de prouver, de s'éprouver dans la voie ouverte par le groupe. Sous cet angle déjà, le sentiment d'appartenance déborde les marges primaires de la simple présence ou de la bonne présentation de soi au groupe. Biologiquement l'action prime, à condition de porter un devenir gratifiant.

La composante intellectuelle et comportementale entre également dans le profil de l'individu pour déterminer sa relation positive au groupe. Ces dernières années ont multiplié les grilles pseudo-théoriques d'accès à la connaissance de soi, d'affirmation de soi par rapport à des «styles» empiriquement définis. On se rangera par exemple à la classification usuelle des styles de négociation ${ }^{10}$ : le factuel (« les faits parlent par euxmêmes »); l'intuitif ("l'imagination peut résoudre tous les problèmes»); le normatif (" négocier c'est marchander »); l'analytique ( la logique convient toujours aux bonnes conclusions »). À chacun de s'y retrouver. Sinon les chemins d'accès à soi ne manquent pas. Ici on parle "d'Egostratégies »"11; là il est question "d'Asioscope», comprendre "analyse des pôles de préférence" selon quatre styles: Analyste, Superviseur, Intégrateur, Explorateur ${ }^{12}$. Ailleurs on se reconnaîtra peut-être dans les styles de communication explorés par Virginia Satir ${ }^{13}$ : agressif ( mon opinion et mes goûts, ou rien »); apaisant («pauvre moi : je suis sans personnalité »); intellectuel («je n’ai pas d'opinion ni de goût vraiment personnel mais je suis l'avis intellectuel des autres»!); manipulateur («j'aime me faire prier un peu») ; affirmatif (« Voici ce que je veux »). Si le sujet se croit étranger à ces premières taxinomies, qu'il persévère! À force de bonne volonté il identifiera, sous une forme ou une autre, "son " style, "son» domaine de référence. Impossible d'échapper à la catégorisation, tant s'exerce sur le marché de l'édition la vague d'inspiration nominaliste. En retour ces classifications empiriques ont le mérite d'offrir aux GRH (lire "Gestionnaires des Ressources Humaines») des tableaux « d'identification » commodes, en vision rationnelle et simplifiée.

Avec sa méthode des « socio-styles » la psycho-sociologie prônée par Bernard Cathelat ${ }^{14}$ s'inscrit quelque peu dans cette continuité. Mais ici le modèle prend de l'ampleur, le "système " investit des champs d'analyse de plus en plus ambitieux, au plan social, professionnel, culturel. D'où sa séduction pour toute circonstance où la définition d'un style de vie propre est en jeu. Indépendamment des déterminations sociales affectant l'habitus de classe du sujet, il lui est donné de se situer sur une « cartographie » sociale des mentalités (activiste, rigoriste, matérialiste, décalage, égocentrage). Le lieu ne se prête pas ici à un développement quelconque de ce modèle, ni à une discussion sur ses limites. Cela nous éclaire toutefois sur la profusion des systèmes descriptifs, voire explicatifs, donnant à la personnalité un cadre de référence, la rationalité d'un découpage, imaginairement vécu comme un alibi pour prendre place dans la mosaïque de l'organisation.

21 Sous l'angle des valeurs vient s'adjoindre, à la composante sociale de l'homme multidimensionnel, un invariant relationnel. Là encore de modèles interactionnistes ${ }^{15}$ en programmation neuro-linguistique ${ }^{16}$ se dessinent des cadres de pensée séduits par de nouveaux miroirs. Qu'importe parfois si les vulgates déplacent le modèle original ou déforment son objet. L'essentiel de toutes ces grilles conceptuelles est de penser un mode d'être en organisation, légitime et valorisant. Dans la relation à autrui le sujet trouve, par identification, les moyens de se nommer. En cas contraire, c'est-à-dire en méconnaissance des modèles évaluatifs retenus par l'organisation, le sujet s'offre à l'économie de ces modèles, avec la virtualité de correspondre puis de s'insérer au cadre d'origine. 
22 Au plus profond de la dimension de soi, orientée vers cette organisation, opère un discours à charge plus symbolique encore. Les types d'approche les plus répandus, comme l'analyse transactionnelle, se démarquent de la psychanalyse. L'individu accède alors sans difficulté à des notions productives comme les états du moi, les positions de vie, les modalités d'échanges et les transactions. Avec les théories de Palo Alto ${ }^{17}$, il ne s'agit pas moins de s'en tenir à un niveau thérapeutique de la communication, pour en corriger les symptômes, recadrer constamment les représentations du réel. Rendre l'individu conscient de sa subjectivité, l'orienter pour calibrer le monde extérieur, en situation de métacommunication, voilà de nouvelles voies tracées dans la relation ouverte à l'environnement. À suivre ces modèles, on avancerait sans doute dans la compréhension des points de motivation à la base du sentiment d'appartenance. Beaucoup d'auteurs s'arrêtent cependant à l'analyse des besoins comme satisfaits de parvenir à une classification homogène, d'allure exhaustive. Donnons, à titre indicatif, l'échelle de R. Francès ${ }^{18}$ redondante avec la majorité des inventaires dressés à ce sujet :

- indépendance de pensée, d'action

- réalisation de soi

- participation aux méthodes

- sentiment d'être informé

- sentiment d'estime dans la position

- développement de soi

- participation aux buts et aux objectifs

- sentiment de prestige

- autorité attachée à la position

- occasion de développer des relations

Sandra Michel ${ }^{19}$ montre néanmoins comment la séquence motivationnelle déborde le cadre étriqué du besoin. Étudiée dans sa complexité la motivation apparaît comme le facteur clé de l'intégration au groupe, par une logique structurante de buts (pratiques), de scénario (itinéraire symbolique), de désirs (impossibles à combler), d'aspirations et d'intentions d'agir ${ }^{20}$. Cette conception dynamique, énergétique de la motivation, relance, avec le corrélat positif du sentiment d'appartenance, le schème de l'identité.

Le parallèle établi jusqu'ici entre l'organisation et le sujet aura insisté avec force sur l'émergence de valeurs non utilitaires comme condition de reconnaissance mutuelle. Dans leur incapacité à mobiliser les énergies, les données économiques de rentabilité, de profit, de productivité, se réinscrivent dans le discours mythique de l'organisation. Le nouveau paradigme des entreprises communicantes décline à l'unisson les mots phares de l'accès à soi et au monde : défi, compétition, challenge. Nous le savons pour l'avoir déjà souligné. Mais nous en arrivons maintenant à des prolongements utiles.

En effet le sens de la compétition, amplifié par les médias, est si récurrent dans les esprits qu'il modifie toutes les données de la communication. Les valeurs ainsi portées par l'entreprise, en stratégie externe, affectent le système même de représentation de l'individu dans son accès au groupe. S'il se reconnaît dans l'âme conquérante de la chevalerie d'entreprise, il aura pour mission d'en sauvegarder la légitimité contre toutes les atteintes possibles: attaques des concurrents, contraintes du marché et même tentatives d'OPA face auxquelles il faudra serrer les rangs, apporter tout le soutien nécessaire. Cette implication prend la forme d'une intériorisation des valeurs données comme universaux conceptuels. 

comme hostiles ou agressives par rapport à l'âme du groupe. $Y$ compris dans les manifestations internes comme les revendications syndicales ou dans les rôles parasites (freinage, défaitisme, lobbysme) qui voudraient nuire à l'image de ce même groupe. Car il s'agit bien d'image. C'est l'un des grands bouleversements de notre société de voir l'eschatologie d'entreprise et la modélisation de son discours, se substituer aux grands systèmes interprétatifs (l'école, la nation, l'église, la cité) qui n'offrent plus de réponses cohérentes. Or, le propre d'une image c'est de produire du sens. En reléguant la logique marchande derrière un discours, des valeurs, un espace de communication fondamental, l'entreprise construit, du réel aujourd'hui diffus, une image rassurante de principes et de convictions inébranlables. Un ethos, c'est-à-dire une morale pratique, et un telos, une finalité à l'action. consensuelles dont l'idio-syncrasie menace l'équilibre vital du groupe. Le revers de cette attitude n'en demeure pas moins le risque d'allégeance parfois aussi servile qu'inconscient à des modèles dominants. En croyant gagner sa liberté dans l'éthique de la compétition, du challenge, le sujet s'abîme dans une course aux chiffres, aux points, aux indices. Il mesure tous les autres à l'aune de sa motivation, les juge, les pèse, regarde en un mot s'ils font le poids dans un tel contexte. Lui-même cautionne et pérennise le système d'évaluation, de régulation, s'auto-contrôle en principe au nom de la transparence mais plus sûrement au service d'une visibilité profitable à l'organisation.

Il arrive cependant que l'adhésion au groupe soit feinte. C'est le cas où le salarié utilise l'entreprise au service de ses propres finalités sans avoir à se demander si le régime de son action obéit à la sincérité. En l'occurrence l'entreprise fournit involontairement le cadre d'une action égoïste, peu scrupuleuse de son calcul avec les valeurs du groupe. Cela revient à opérer, sans états d'âme, sur un champ d'opérations à la limite artificiel mais ô combien stimulant, utile à exploiter.

29 À des niveaux de motivation moindres la réaction identitaire subsiste chez l'individu disposé à se ranger dans la logique du système. À défaut de conviction, en méconnaissance de ses aspirations ou désirs profonds, il vit son appariement au groupe par immersion et habitude. On lui demandera au besoin de s'impliquer dans des actions de base, sans faire nécessairement appel à des motivations plus intenses. Cette version instrumentale de l'appartenance au groupe ouvre le champ à des techniques de management directives, voire autocratiques, pour substituer à l'image charismatique du leader le poids plus concret d'une autorité.

30 À travers tous ces changements d'angles, le schème identitaire recouvre par conséquent des réalités disparates, parfois contradictoires. Tantôt les valeurs de l'entreprise s'incarnent dans le sujet qui en acquiert raison d'être et reconnaissance. Tantôt ces valeurs s'imposent du dehors et déterminent le sujet soit à les exploiter artificiellement, soit à les accepter sur le mode endoxal, comme un système allant de soi, avec des normes pourtant inculquées. Cette vocation pédagogique de l'organisation gagne malgré tout à maintenir un cadre démocratique. Ne serait-ce que dans l'éthique des Relations Humaines pour valider, par un dialogue renoué, les formes ajustées du discours dominant. ${ }^{21}$

31 Une autre façon de considérer le problème consiste à étudier comment le sentiment d'appartenance se nourrit d'une relation mimétique profonde entre le sujet et l'organisation. Non plus en termes psychologiques d'identification, d'intégration active 
ou passive mais dans le paradigme de «l'action communicante » (Habermas), de « l'action collective» (Crozier). Se réalise alors le passage fondamental d'une conception organiciste et linéaire, divisée en deux camps, à une vision organisationnelle, stratégique, ouverte au jeu de l'interaction. Elle recadre surtout le sentiment d'appartenance dans une perspective systémique, comme "attitude émergente» (J. de Rosnay) propice à donner corps au terme d'organisation et vie au concept de système.

Il est banal de souligner combien la relation au travail est aujourd'hui conditionnée par les paramètres de l'organisation. Des processus de production à la gestion des ressources humaines, par la médiation des règles et procédures, le groupe devient à plein sens du terme une « organisation ». S'il importe d'en saisir la «structure » et la "dynamique »"2, il apparaît non moins utile de distinguer, au plan de l'individu, les bases du processus motivationnel: "Plus le salarié considère que les buts et projets de l'entreprise coïncident avec les siens propres, plus il mettra au service de l'entreprise le dynamisme inhérent au processus motivationnel $»^{23}$. Nous savons que cette motivation s'inscrit dans une séquence où, en dehors des buts et projets, le sujet doit faire l'expérience de choix, manifester des intentions d'agir à l'intérieur d'un processus global et non simplement par vision instrumentale (compensation financière, évaluation concrète d'un type de récompense). En particulier les objectifs n'incombent pas à l'individu mais à l'équipe, avec en retour une vision stratégique réappropriée par le sujet, dans un champ défini.

Les divisions classiques du management (autocratique, démocratique, participatif) marquent alors le pas face à une nouvelle éthique, dans l'esprit de «l'action comunicative » décrite par Habermas ${ }^{24}$. C'est-à-dire une action ayant comme fin l'entente, comme principe l'intercompréhension et comme moyen l'échange d'arguments rationnels. L'action communicative se place ainsi au cœur du management dit « éducatif » qui, comme tout système de management adapté à notre époque, crée de la valeur ajoutée avec un élément de base : l'homme. Le modèle du management éducatif ne heurte pas les formes traditionnelles comme le management participatif (action stratégique) ou le management par le commandement (action instrumentale). Il les subsume dans un contexte où le sujet puisse vraiment acquérir sa dignité et s'orienter vers l'entente, en vertu d'une action franche mais concertée, d'une communication non conciliante par nature mais par volonté de compréhension.

Pourtant, rapporté à l'organisation, ce modèle aussi séduisant soit-il, relève de conceptions encore abstraites (symbolique du lien social), de données implicites (l'horizon culturel), par définition difficiles à objectiver ou formaliser. Il faut alors se tourner vers Michel Crozier et le Centre de Sociologie des Organisations pour éclairer notre problématique du jeu entre "l'acteur et le système $»^{25}$. La thèse fondamentale est qu'au sein de l'organisation, le sujet se présente comme un agent rationnel mais libre, ayant un pouvoir d'action stratégique sur plusieurs "tableaux», en fonction d'opportunités mises en jeu au sein du système.

Le terme d'opportunités ne doit pas faire penser à une démarche purement individualiste, déjà entrevue ici. L'action stratégique organise des modes d'intégration et d'action collective pour garantir la coopération des acteurs : «Les construits d'action collective dans leurs différentes modalités constituent la solution. Par eux, les problèmes sont redéfinis et les champs d'interaction aménagés ou « organisés » de telle façon que dans la poursuite de leurs intérêts spécifiques les acteurs ne mettent pas en danger les résultats de l'entreprise collective, voire les améliorent. Bref, ils organisent des modes d'intégration qui assurent la nécessaire coopération entre acteurs sans supprimer leurs 
libertés, c'est-à-dire leur possibilité de poursuivre des objectifs contradictoires ${ }^{26}$. Les construits d'action collective ont donc cette propriété de réguler, d'harmoniser les jeux organisationnels en respect de l'intégrité individuelle. L'adhésion au groupe ne subit pas l'empreinte totalitaire du "même", ni la menace du «tautisme » où Lucien Sfez ${ }^{27}$ voit une cristallisation de l'autisme, de la tautologie et du totalitarisme. Avec l'approche stratégique le sujet s'affirme, au contraire, comme lucide et disponible à son environnement, pour conquérir sa liberté.

Il ne faut voir dans l'éducation systémique que la confirmation de cette tendance, avec notamment des principes de base comme la causalité mutuelle, l'interdépendance, le primat de la relation sur le contenu. Nous reprenons ici, en la simplifiant, la dichotomie proposée par Joël de Rosnay ${ }^{28}$ entre "l'attitude traditionnelle» et «l'attitude émergente ». L'opposition décrite correspond globalement à une relation de contrariété entre la conception dualiste (traditionnelle) de l'organisation et une conception stratégique (émergente) prônant l'interaction des éléments du système :

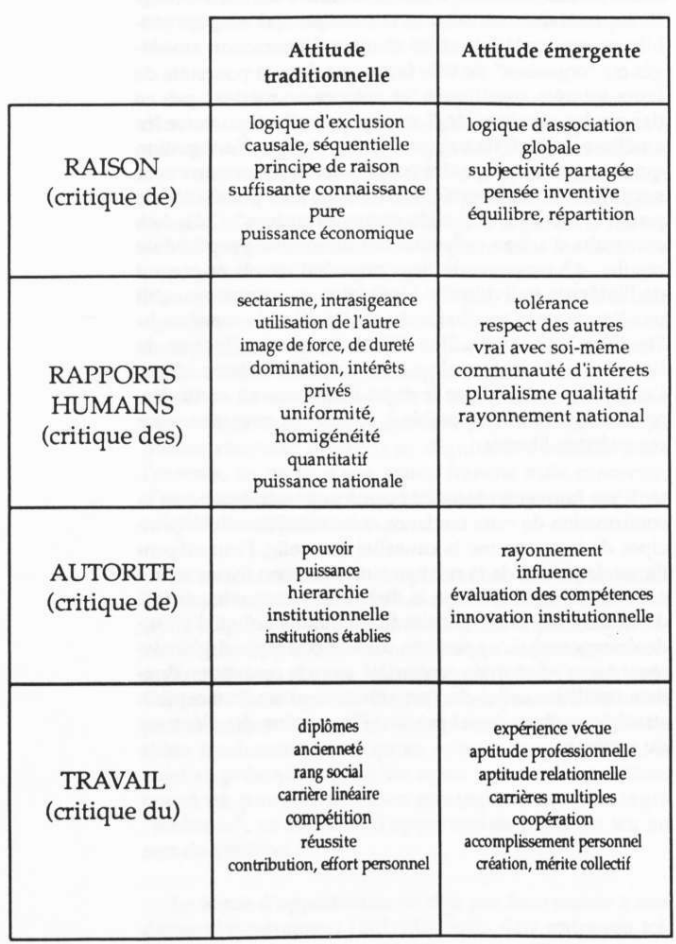

Il est évident qu'à l'intérieur du système la "subjectivité partagée » entre soi et les différents acteurs représente, pour le sujet, un espace de construction valorisant où le sentiment d'appartenance au groupe se forge une identité durable et sincère, épanouissante. Mais de besoin en attachement, d'implication en motivation, de réalisation de soi en performance, l'affirmation identitaire ne se laisse guère appréhender, en organisation, sur le seul mode empirique des attitudes et des comportements.

Le rôle de la communication est de réinscrire cette dimension psychologique à travers un processus global de signification, c'est-à-dire à travers des formes de discours et de représentations. Une recherche de ce type donnerait à notre propos son prolongement véritable, en reconnaissant dans l'infinité des situations possibles ${ }^{29}$ selon des paramètres économiques, sociaux, culturels, des éléments structurant d'une grande stabilité. La 
nécessité d'abord pour l'organisation de bâtir et de promouvoir une image congruente avec sa philosophie et son action. La nécessité corrélative pour le sujet de définir son image sous le double rapport de l'affirmation de soi et de l'expression non seulement d'un " profil » mais d'une personnalité dans un cadre de référence à conceptualiser.

Il apparaît que le sujet comme l'organisation peuvent se rencontrer, confondre leurs énergies, leur sens des responsabilités et de l'innovation. Cependant, à l'écart des modèles systémiques et stratégiques, le sentiment d'appartenance s'appuie au besoin sur des schèmes plus réducteurs. Les valeurs utilitaires bien sûr, mais aussi les Relations Humaines quand elles servent d'alibi à un mode d'intériorisation plus souple du discours dominant, parfois sur un mode artificiel.

Depuis Taylor et Fayol, un pas considérable a été franchi pour la reconnaissance de l'homme comme valeur ajoutée dans l'organisation, pour l'épanouissement d'un sentiment d'identité avec le groupe, voire une nouvelle "logique de l'honneur » (30). Mais d'intentions en déclarations, de communication en métacommunication, gardonsnous de substituer au discours machinique, la mécanique d'un discours tout aussi pervers dans ses effets.

\section{NOTES}

1. FAYOL H., Administration industrielle et générale, Dunod, Paris, 1979.

2. TAYLOR F. W, La direction scientifique des entreprises, Dunod, Paris, 1957.

3. FRIEDMANN G. P., Le travail en miettes, Gallimard, Paris, 1964.

4. MAYO E., The human problems of an industrial civilization, Mac Millan, New York, 1933.

5. M. et P. FAUCHEUX ont traduit et publié quelques articles de Kurt Lewin, in Psychologie dynamique, Presses Universitaires, Paris, 1959.

6. CROZIER M., FRIEDBERG E., L'acteur et le Système Seuil, Paris, 1977.

7. CROZIER M., préface du livre Les Organisations, MARCH \& SIMON, Dunod, Paris, 1979.

8. MASLOW A., Motivation and personnality, Harper, New York, 1954.

9. ROSNAY J. de, Le Macroscope, Points, Seuil, Paris, 1977.

10. CASSE P., DEOL Surinder P.S., La négociation interculturelle, Chotard et Associés, Paris, 1987.

11. CARON G., Egostratégies, Éditions d'organisation, Paris, 1991.

12. DUMONT J., Le temps personnel, Éditions d'organisation, Paris, 1988.

13. SATIR V., Peoplemaking, Science and Behavior Book, Palo Alto, California, 1972.

14. CATHELAT B., Socio-styles système, Éditions d'organisation, Paris, 1990.

15. WINKIN Y., La nouvelle communication, Points, Seuil, Paris, 1984.

16. BANDLER R., GRINDER J., Les secrets de la communication, Le Jour, Paris, 1990.

17. In WINKIM Y., (Bateson, Birdwhistell, Goffman, Hall, Jackson, Scheflen, Sigman, Watzlawick).

18. FRANCES R., Satifaction et motivation chez les cadres, in Quelles motivations au travail ?, Société française de Psychologie, Entreprise moderne d'édition, Paris, 1982.

19. MICHEL S., Peut-on gérer les motivations ? P.U.F., Paris, 1989.

20. SAINSAULIEU R., L'identité au travail, Presse de la fondation nationale des Sciences Politiques, Paris, 1985.

21. MINTZBERG H., Structure et dynamique des organisations, Editions d'organisation, Paris, 1986. 
22. MICHEL S., op. cit. p. 105.

23. HABERMAS J., Théorie de l'agir communicationnel, Fayard, Paris, 1987.

24. CROZIER M., op. cit.

25. Ibid.

26. SFEZ L., Critique de la communication, Seuil, Paris, 1988.

27. ROSNAY J. de, op. cit

28. DARBELET M., Économie d'entreprise, Foucher, Paris, 1987.

29. IRIBARNE P., La logique de l'honneur, Seuil, Paris, 1989.

\section{RÉSUMÉS}

Le sentiment d'appartenance en organisation peut s'appréhender selon différents modes d'accès : psychologique, sociologique, historique, etc. Mais la recherche d'une cohérence confère à l'approche en communication une valeur heuristique originale et productive sur le sujet. L'identité au groupe renvoie dès lors à un système de significations précis où se recadrent les relations de l'homme et de l'organisation.

\section{AUTEUR}

\section{JEAN-JACQUES BOUTAUD}

Jean-Jacques Boutaud, 38 ans. Maître de Conférences à l'Université de Bourgogne. Enseigne la communication et dirige un programme de sémiotique iconique dans le cadre d'un diplôme européen (DEEM). Travaux en collaboration avec le CRE-LEF (Besançon), EIDOS (Tours) et création d'un pôle de recherche à Dijon (PRISM). Derniers travaux : Entre tout dire et parler guerre (Guerre du Golfe et stratégies discursives), Communication, Québec ; transparence du signe et intention sublimin(ér)ale, Degrés, Bruxelles ; Signes et simulacres du corps : Dali, le Grand Masturbateur, Hispanistica. 